\title{
On Fully-M-Cyclic Modules
}

\author{
Samruam Baupradist (Corresponding author) \\ Department of Mathematics, Faculty of Science \\ Chulalongkorn University, Bangkok 10330, Thailand \\ Tel: 66-83-137-1119 E-mail: samruam.b@chula.ac.th \\ Suphawat Asawasamrit \\ Department of Mathematics, Faculty of Applied Science \\ King Mongkut's University of Technology North Bangkok \\ Bangkok 10800, Thailand \\ Tel: 66-81-346-6321 E-mail: suphawata@kmutnb.ac.th
}

Received: November 26, 2010 Accepted: December 7, 2010 doi:10.5539/jmr.v3n2p23

\begin{abstract}
The aim of this work was to generalize generator, $M$-generated modules in order to apply them to a wider class of rings and modules. We started by establishing a new concept which is called a fully- $M$-cyclic module. We defined this notation by using $\operatorname{Hom}_{R}(M, *)$ operators which are helpful to contract the new construction and describe their properties. Finally, we could see the structure of fully- $M$-cyclic module and quasi-fully-cyclic module by the structure of $M$.
\end{abstract}

Keywords: Fully-M-cyclic modules, Quasi-fully-cyclic modules, Generator modules, Self-generator modules

\section{Introduction}

Throughout this paper, $R$ is an associative ring with identity and $M_{R}$ is the category of unitary right $R$-modules. Let $M$ be a right $R$-module and $S=E n d_{R}(M)$, its endomorphism ring. A right $R$-module $N$ is called $M$-generated if there exists an epimorphism $M^{(I)} \longrightarrow N$ for some index set $I$. If $I$ is finite, then $N$ is called finitely $M$-generated. In particular, $N$ is called $M$-cyclic if it is isomorphic to $M / L$ for some submodule $L$ of $M$. Following Wisbauer [1991], $\sigma[M]$ denotes the full subcategory of Mod- $R$, whose objects are the submodules of $M$-generated modules. A module $M$ is called a self-generator if it generates all of its submodules. $M$ is called a subgenerator if it is a generator of $\sigma[M]$.

\section{On Fully- $M$-cyclic module}

In this part, a module $M$ be given as a right $R$-module.

Definition 2.1. Let $N \in M_{R}$. $N$ is called a fully-M-cyclic module if every submodule $A$ of $N$ is of the form $s(M)$ for some $s$ in $\operatorname{Hom}_{R}(M, N)$.

Remark 2.2. Dealing directly from definition, the following statements are routine:

(1) Submodule of a fully- $M$-cyclic module is a fully- $M$-cyclic module.

(2) If $M$ is simple module and $N$ is fully- $M$-cyclic module, then any nonzero submodule of $N$ is simple submodule.

Definition 2.3. The module $M \in M_{R}$ is called a quasi-fully-cyclic module if it is a fully-M-cyclic module.

Obviously, every semi-simple module is a quasi-fully-cyclic module.

Lemma 2.4. Let $N$ be a fully-M-cyclic module. If $M$ is a noetherian module then $S$ oc $(M) \cong S$ oc $(N)$.

Proof. Since $N$ is a fully- $M$-cyclic module, a simple submodule $B$ of $N$ is of the form $s(M)$ for some $s \in H o m_{R}(M, N)$. By the simply property of $B$, there is $b \in B$ such that $B=b R$. Suppose that $s(a)=b$ for some $a \in M$. In noetherian module $a R$, there exists a simple submodule $A$ containing $a$. It is easily to see that $A \cong B$. Conversely, if $A$ is a simple submodule of $M$ then $s(A)=B$ is a simple submodule of $N$ and then $A \cong B$ for all $s \in \operatorname{Hom}_{R}(M, N)$. This shows that $\operatorname{Soc}(M) \cong \operatorname{Soc}(N)$.

Lemma 2.5. If $N$ is a fully-M-cyclic module then $N$ has no nonzero small submodule.

Proof. In a contrary, we suppose that there is a nonzero submodule $A$ which is small in $N$. Let $B$ be a submodule of $N$ 
such that $A+B=N$. Since $N$ is a fully-M-cyclic module, there are $s, t \in \operatorname{Hom}_{R}(M, N)$ such that $s(M)=A, t(M)=B$. Put $f=s+t$, then $f$ is an epimorphism from $M$ to $N$. Since $A$ is a small submodule of $N, t$ is an epimorphism and hence $s$ is an epimorphism. It follows that $A=N$, a contradiction, showing that $N$ has no nonzero small submodule.

Corollary 2.6. If $N$ is a fully-M-cyclic module then $\operatorname{Rad}(N)=0$.

Definition 2.7. Let $N$ be a fully- $M$-cyclic module. For a submodule $A$ of $N$ there exists a homomorphism $s \in \operatorname{Hom}_{R}(M, N)$ such that $s(M)=A$. $s$ is called a presented homomorphism of $A$.

Lemma 2.8. Let $N$ be a fully-M-cyclic module. If $s$ is a presented homomorphism of a submodule $A$ of $N$ then $A$ is maximal if and only if every $t \in S=\operatorname{Hom}_{R}(M, N)$ with $\operatorname{Im}(t)$ containing the image of presented homomorphism of $A$ is an epimorphism.

Proof. Let $A=s(M) \subset_{>} \operatorname{Im}(t)$ in $N$. Since $A$ is a maximal submodule of $N$ then $\operatorname{Im}(t)$ must be $N$, and hence $t$ is an epimorphism. Conversely, let $A=s(M)$ and $A \subset_{>} B$. Since $N$ is a fully- $M$-cyclic module, there is an element $t \in \operatorname{Hom}_{R}(M, N)$ such that $B=t(M)$. By assumption, the non equality $s(M) \subset_{>} t(M)$ follows that $t$ is an epimorphism, and hence $B=N$.

Leading directly from definition, the following properties in Lemma 2.9 are routine,

Lemma 2.9. Let $N$ be a fully-M-cyclic module and $A$ be a submodule of $N$ and s its a presented homomorphism.

(1) If $M$ is an epimorphism image of $M^{\prime}$ then $N$ is also a fully- $M^{\prime}$-cyclic module.

(2) If $M$ is a fully- $M^{\prime}$-cyclic module then $N$ is also a fully- $M^{\prime}$-cyclic module.

(3) $A$ is an essential in $N$ if and only if for any nonzero element $t$ of $\operatorname{Hom}_{R}(M, N), \operatorname{Im}(t) \cap \operatorname{Im}(s) \neq 0$.

(4) $A$ is uniform if and only if every $t \in \operatorname{Hom}_{R}(M, N)$ with $0 \neq \operatorname{Im}(t) \subset_{>} \operatorname{Im}(s)$ then $\operatorname{Im}(t)$ is an essential in $\operatorname{Im}(s)$.

(5) $A$ is a direct summand of $N$ if and only if there exists $t \in \operatorname{Hom}_{R}(M, N)$ such that $\operatorname{Im}(s) \cap \operatorname{Im}(t)=0$ and $s+t$ is an epimorphism.

\section{Quasi-fully-cyclic module}

In this part, we put $S=\operatorname{End}_{R}(M)$. We have known that for any right $R$-module $M$, the $\operatorname{direct}$ summand $A$ of $M$ is image of a presented homomorphism which is an idempotent of $S$ but not all. Which is case of the form submodules such that every its presented homomorphisms are idempotents?. The following lemma is a clear answer:

Lemma 3.1. Let $M$ be a quasi-fully-cyclic module. If $A$ is a simple submodule of $M$ with $s$ its a presented homomorphism then $s$ is an idempotent of $S=\operatorname{End}_{R}(M)$.

Proof. Let $s$ be a presented homomorphism of $A$. Because $A$ is a simple submodule of $M$ then $s^{2}(A) \neq 0$. Therefore, we have $0 \neq s^{2}(M) \subset_{>} s(M)=A$ and $s^{2}(M)$ must be equal to $A=s(M)$, showing that $s$ is an idempotent of $S$.

Right now, we suppose that $M$ be a quasi-fully-cyclic module. If $e^{2}=e$, the one gets a direct sum decomposition $M=e(M) \oplus(1-e)(M)$. Conversely, if $M=A \oplus B$ then we can write $1=\pi_{A}+\pi_{B}$ with $\pi_{A}$ (resp. $\pi_{B}$ ) being a natural projection map from $M$ to $A$ ( resp. $B$ ). $\pi_{A}\left(\operatorname{resp} . \pi_{B}\right.$ ) is an idempotent element of $S$ which is a presented homomorphism of $A$ ( resp. $B$ ) so that we can get the following corollary.

Corollary 3.2. In a quasi-fully-cyclic module, every simple submodule is a direct summand.

Theorem 3.3. Let $M$ be a quasi-fully-cyclic module. $M$ is a Noetherian (resp. Artinian) if and only if $S$ is a right self Noetherian (resp. Artinian) ring.

Proof. Suppose that $M$ is Noetherian. We may easily analogize our self the proof of the case Artinian. Take any ascending chain of the right ideals $s_{1} S \subset_{>} s_{2} S \subset_{>} s_{3} S \subset_{>} \ldots s_{n} S \subset_{>} \ldots$ of the ring $S$. Since $s_{i} S \subset_{>} s_{i+1} S, s_{i}=s_{i+1} t$ for some $t \in S$. We have $s_{i}(M) \subset_{>} s_{i+1}(M)$ for all $i \in N$. The ascending chain of the submodules $s_{1}(M) \subset_{>} s_{2}(M) \subset_{>}$ $s_{3}(M) \subset_{>} \ldots \subset_{>} s_{n}(M) \subset_{>} \ldots$ must be stationary in the noetherian module $M$ so that $s_{n_{0}}(M)=s_{n_{0}+j}(M)$ for some $n_{0}$ and all $j \geq 0$. This implies that for $i \geq n_{0}$ there is a permutation function $t \in S$ such that $s_{i+1}=s_{i} t$ for some $t \in S$. It follows that $s_{i+1} S \subset_{>} s_{i} S$, and hence $s_{i+1} S \subset_{>} s_{i} S$ for all $i \geq n_{0}$. It says that the ascending chain of the right ideals $s_{1} S \subset_{>} s_{2} S \subset_{>} s_{3} S C_{>} \ldots s_{n} S \subset_{>} \ldots$ must be exact stationary at $n_{0}$. Conversely, if $S$ is a right self noetherian ring. Take any ascending chain of the submodules $A_{1} \subset_{>} A_{2} \subset_{>} A_{3} \subset_{>} \ldots \subset_{>} A_{n} \subset_{>} \ldots$ of $M$. Since $M$ is a quasi-fully-cyclic module, for every $i$ index, there is $s_{i} \in S$ such that $s_{i}(M)=A_{i}$. Following that $s_{1} S \subset_{>} s_{2} S \subset_{>} s_{3} S \subset \subset_{>} \ldots s_{n} S \subset \subset_{>} \ldots$ is a ascending chain of the right ideals of $S$. By assumption, this ascending chain must be stationary at some $n_{0}$ index. 
Therefore, $s_{i} S=S_{i+1} S$ for all $i \geq n_{o}$. This shows that $s_{i}(M)=s_{i+1}(M)$ for all $i \geq n_{0}$. And hence the given ascending chain of the submodules $A_{1} \subset_{>} A_{2} \subset_{>} A_{3} \subset_{>} \ldots \subset_{>} A_{n} \subset_{>} \ldots$ is stationary at $n_{0}$. The proof now is completed.

Lemma 3.4. For each quasi-fully-cyclic-module, the following statements are equivalent:

(1) $S$ is artinian;

(2) $M$ is finitely co-generated;

(3) $M$ is semisimple and finitely generated;

(4) $M$ is semisimple and noetherian;

(5) $M$ is the direct sum of a finite set of simple submodules.

Proof. We refer to the ([Anderson, 1974], Proposition 10.15) for the proving of $3 \Longleftrightarrow 4 \Longleftrightarrow 5$. By the Theorem 3.3, we know that $S$ is artinian if and only if $M$ is artinian. By the Corollary 2.6, we have $\operatorname{Rad}(M)=0$. The proof is now completed by turning back to apply the ([Anderson, 1974], Proposition 10.15).

Definition 3.5. Let $M$ be a right $R$-module. $M$ is called Hopfian (resp. co-Hopfian) if every surjective (resp. injective) endomorphism of $M$ is an automorphism.

Definition 3.6. Let $M$ be a right $R$-module. $M$ is called a Fitting module if every endomorphism $f$ of $M$ satisfies Fitting's lemma (i.e. there exists an integer $n \geq 1$ such that $M=\operatorname{Ker}\left(f^{n}\right) \oplus \operatorname{Im}\left(f^{n}\right)$ ).

Lemma 3.7. Let $M$ be a quasi-fully-cyclic-module. If $M$ is finitely cogenerated and Hopfian then for any $s \in S$ there exists an integer number $n$ such that $M=\operatorname{Ker}\left(s^{n}\right) \oplus \operatorname{Im}\left(s^{n}\right)$.

Proof. Since $M$ is both a quasi-fully-cyclic and finitely cogenerated and by the Lemma 3.4, we have $M$ is artinian. Applying the ([Anderson, 1974], Lemma 11.6) to the Hopfian module $M$, we have $M$ is a Fitting module. This shows that for any $s \in S$ there exists an integer number n such that $M=\operatorname{Ker}\left(s^{n}\right) \oplus \operatorname{Im}\left(s^{n}\right)$.

Theorem 3.8. Let $M$ be a quasi-fully-cyclic module.

(1) For any $s, u \in S, l_{S}(\operatorname{Im}(u))+S s \subset_{>} l_{S}(\operatorname{Im}(u) \cap \operatorname{Ker}(s))$.

(2) If $N$ is a maximal submodule of $M$ then $l_{S}(N)$ is a minimal left ideal of $S$.

Proof. (1) According to the relationship $\operatorname{Im}(u) \cap \operatorname{Ker}(s) \subset_{>} \operatorname{Im}(u)$ follows that $l_{S}(\operatorname{Im}(u)) \subset_{>} l_{S}(\operatorname{Im}(u) \cap \operatorname{Ker}(s))$. Take any $t s \in S s$ and $m \in \operatorname{Im}(u) \cap \operatorname{Ker}(s)$. We have $t s(m)=0$. It implies that $t s \in l_{S}(\operatorname{Im}(u) \cap \operatorname{Ker}(s))$, and hence $S s \subset>$ $l_{S}(\operatorname{Im}(u) \cap \operatorname{Ker}(s))$. Therefore, $l_{S}(\operatorname{Im}(u))+S s \subset_{>} l_{S}(\operatorname{Im}(u) \cap \operatorname{Ker}(s))$.

(2) Since $M$ is quasi-fully-cyclic module, there exists $s_{0} \in S$ such that $s_{0}(M)=N$. Therefore, $l_{S}(N)=\left\{t \in S \mid t s_{0}=0\right\}$. It is easy to see that $l_{S}(N)$ is one of the form of left ideals of $S$. Take any $0 \neq t \in l_{S}(N)$ then $t(N)=0$ saying that $N \subset_{>} \operatorname{Ker}(t)$. By maximality of $N, \operatorname{Ker}(t)$ is $N$. Right now, if we take any $k \in l_{S}(N), k(N)=0$ shows that $\operatorname{Ker}(t) \subset \operatorname{Ker}(k)$. It follows that there is $s \in S$ such that $k=s t$, and hence $k \in S t$. Thus it is $l_{S}(N) \subset S t$, and hence $l_{S}(N)=S t$, showing minimality of $l_{S}(N)$.

\section{Acknowledgment}

The authors would like to thank Dr. Hong Dinh Hai for his encouragement and suggestion. This paper is supported by King Mongkut's University of Technology North Bangkok, Thailand.

\section{References}

Anderson, F. W. \& Fuller, K. R. (1974). Rings and Categories of Modules, Graduate Texts in Math. No.13, SpringerVerlag, New York, Heidelberg, Berlin.

Camillo, V. \& Yousif, M. F. (1991). Continuous rings with ACC on annihilators. Cand. Math. Bull., 34, $642-644$.

Dung, N. V., Huynh, D. V., Smith, P. F. \& Wisbauer, R. (1994). Extending Modules. Research Notes in Mathematics Series, 313, Pitman London.

Faith, C. (1966). Rings with ascending chain condition on annihilators. Nagoya Math. J., 27, 179-191.

Jain, S. K., Singh, S., \& Symonds, R. G. (1976). Rings whose proper cyclic modules are quasi-injective. Pacific J. Math, 67, 461-472.

Kasch, F. (1977). Moduln and Ringe, Stuttgart. 
Mohamed, S. H. \& Müller, B.J. (1990). Continuous and Discrete Modules. London Math. Soc. Lecture Note Series, No. 147, Cambridge Univ. Press.

Nicholson, W. K. \& Yousif.M. F. (1995). Principally injective rings. Journal of Algebra, 174, 77-93.

Sanh, N. V., Shum, K. P., Dhompongsa, S. \& Wongwai, S. (1999). On quasi-principally injective modules. Algebra Colloquium, 6:3, 269-276.

Wisbauer, R. (1991). Foundations of Module and Ring Theory. Gordon and Breach, London, Tokyo, e.a. 